\title{
Electronic Correlations within Fermionic Lattice Models
}

\author{
M. MAtlak ${ }^{a, *}$, B. Grabiec ${ }^{b}$ And S. KrawieC ${ }^{a}$ \\ ${ }^{a}$ Institute of Physics, University of Silesia \\ Uniwersytecka 4, 40-007 Katowice, Poland \\ ${ }^{b}$ Institute of Physics, University of Zielona Góra \\ Prof. Z. Szafrana 4a, 65-516 Zielona Góra, Poland
}

(Received July 10, 2007)

\begin{abstract}
We investigate two-site electronic correlations within generalized Hubbard model, which incorporates the conventional Hubbard model (parameters: $t$ (hopping between nearest neighbours), $U$ (Coulomb repulsion (attraction)) ) supplemented by the intersite Coulomb interactions (parameters: $J^{(1)}$ (parallel spins), $J^{(2)}$ (antiparallel spins)) and the hopping of the intrasite Cooper pairs (parameter: $V$ ). As a first step we find the eigenvalues $E_{\alpha}$ and eigenvectors $\left|E_{\alpha}\right\rangle$ of the dimer and we represent each partial Hamiltonian $E_{\alpha}\left|E_{\alpha}\right\rangle\left\langle E_{\alpha}\right|(\alpha=1,2, \ldots, 16)$ in the second quantization with the use of the Hubbard and spin operators. Each dimer energy level possesses its own Hamiltonian describing different two-site interactions which can be active only in the case when the level will be occupied by the electrons. A typical feature is the appearance of two generalized $t-J$ interactions ascribed to two different energy levels which do not vanish even for $U=J^{(1)}=J^{(2)}=V=0$ and their coupling constants are equal to $\pm t$ in this case. In the large $U$-limit for $J^{(1)}=J^{(2)}=V=0$ there is only one $t-J$ interaction with coupling constant equal to $4 t^{2} /|U|$ as in the case of a real lattice. The competition between ferromagnetism, antiferromagnetism and superconductivity (intrasite and intersite pairings) is also a typical feature of the model because it persists in the case $U=J^{(1)}=J^{(2)}=V=0$ and $t \neq 0$. The same types of the electronic, competitive interactions are scattered between different energy levels and therefore their thermodynamical activities are dependent on the occupation of these levels. It qualitatively explains the origin of the phase diagram of the model. We consider also a real lattice as a set of interacting dimers to show that the competition between magnetism and superconductivity seems to be universal for fermionic lattice models.
\end{abstract}

PACS numbers: 71.10.--w, 71.10.Ca, 71.10.Fd, 71.20.Be, 71.27.+a, 71.28.+d, 73.21.La, 78.67.Bf

*corresponding author; e-mail: matlak@us.edu.pl 


\section{Introduction}

Model Hamiltonians which serve to describe electronic subsystems of solids are formulated on the quantum mechanical basis which takes into account interaction processes between electrons, most of them basing on the Hubbard model and its generalizations (cf. e.g. Refs. [1-96]). The ground state properties of such models has been investigated in Refs. [85-92]. Each model Hamiltonian of this type describes, however, as a rule many unknown, competitive, electronic correlations (two-site, three-site, etc.) which are normally invisible in the original model but the knowledge about their existence is very important because just these correlations determine also an area of physics where a given model Hamiltonian can really be applied. To find the electronic correlation within a given model Hamiltonian $H$ let us assume that we can exactly solve the Schrödinger equation $H\left|E_{\alpha}\right\rangle=E_{\alpha}\left|E_{\alpha}\right\rangle$. Having to our disposal the calculated eigenvalues $E_{\alpha}$ and eigenvectors $\left|E_{\alpha}\right\rangle$ we can use the equivalent form of the given Hamiltonian $H=\sum_{\alpha} H_{\alpha}$ where the set of commuting partial Hamiltonians $H_{\alpha}=E_{\alpha}\left|E_{\alpha}\right\rangle\left\langle E_{\alpha}\right|$ can be represented in the second quantization for each energy level separately introducing here Hubbard and spin operators. In this way to each energy level of the system $E_{\alpha}$ a partial Hamiltonian $H_{\alpha}$ can be ascribed. Each part $H_{\alpha}$ contains many important, competitive interactions, active in the case when the level will be occupied by the electrons. This simple idea cannot be unfortunately applied in a general case because we cannot exactly solve the mentioned Schrödinger equation. It is, however, possible to do it exactly and analytically in the case of a dimer described by the generalized Hubbard model (see Sect. 2) to show that two-site electronic correlations resulting from this approach describe the competition between magnetism and superconductivity. We show that this competition is a universal feature of all electronic lattice models containing hopping term and it takes also place in the case of a real lattice. This result seems to be very important also in the case of quantum dots and nanostructures (cf. e.g. Refs. [97-100]).

\section{The model}

The generalized one-band Hubbard model belongs to a class of fermionic lattice models widely used in the solid state physics. This model has been primarily applied to explain magnetic and transport properties of transition metals, their compounds and alloys, including also insulator-metal transitions (cf. e.g. Refs. [1-16] and original papers cited therein). After further generalizations the model has also been applied to fluctuating valence systems and heavy fermions (Anderson-like models, Refs. [17, 18] (see also Ref. [13] for a review)), liquid ${ }^{3} \mathrm{He}$ (see e.g. Refs. [19-21]) and fullerenes (cf. e.g. Refs. [22-24]). A special attention has been, however, paid in recent decades to the theory of high- $T_{\mathrm{C}}$ superconductivity (cf. Refs. [25-58]) where the extended Hubbard model (negative $U$ model) has widely been used (see e.g. Ref. [33] for a review). Another interesting model also used in the context 
of superconductivity is the Kulik-Pedan-Penson-Kolb (KPPK) model, formulated in Refs. [59, 60] (see also e.g. Refs. [61-64]). In the present paper we consider the extended Hubbard model supplemented by the hopping of the intrasite Cooper pairs (KPPK interaction). The Hamiltonian of this model has the form

$$
\begin{aligned}
H= & \sum_{i \neq j, \sigma} t_{i, j} c_{i, \sigma}^{+} c_{j, \sigma}+U \sum_{i} n_{i, \uparrow} n_{i, \downarrow}+\frac{1}{2} \sum_{i \neq j, \sigma} J_{i j}^{(1)} n_{i, \sigma} n_{j, \sigma} \\
& +\frac{1}{2} \sum_{i \neq j, \sigma} J_{i j}^{(2)} n_{i, \sigma} n_{j,-\sigma}-\sum_{i \neq j} V_{i, j} c_{i, \uparrow}^{+} c_{i, \downarrow}^{+} c_{j, \downarrow} c_{j, \uparrow} .
\end{aligned}
$$

The indices $(i, j)$ enumerate the lattice points $\left(\boldsymbol{R}_{i}, \boldsymbol{R}_{j}\right), t_{i, j}$ is the hopping integral, $U$ denotes the effective intrasite Coulomb interaction, $J^{(1)}$ and $J^{(2)}$ (generally, not necessary equal) describe the effective intersite interactions, all of them resulting from the original intrasite and intersite Coulomb repulsion which can be modified by polaronic effects (see e.g. Ref. [33] for details) and therefore $U, J^{(1,2)}$ can be treated here as positive or negative parameters. The last term in (1) is responsible for the transport of the intrasite Cooper pairs (Refs. [59, 60]) with the coupling constant $V$. The model (1) cannot be solved exactly in a general case. We can, however, consider a special but nontrivial case of two interacting ions (a dimer problem) which possesses an exact, analytical solution. Thus, let us start with the dimer Hamiltonian, resulting from the expression (1). It has the form

$$
\begin{aligned}
H_{\mathrm{D}}= & -t \sum_{\sigma}\left(c_{1, \sigma}^{+} c_{2, \sigma}+c_{2, \sigma}^{+} c_{1, \sigma}\right)+U\left(n_{1, \uparrow} n_{1, \downarrow}+n_{2, \uparrow} n_{2, \downarrow}\right)+J^{(1)} \sum_{\sigma} n_{1, \sigma} n_{2, \sigma} \\
& +J^{(2)} \sum_{\sigma} n_{1, \sigma} n_{2,-\sigma}-V\left(c_{1, \uparrow}^{+} c_{1, \downarrow}^{+} c_{2, \downarrow} c_{2, \uparrow}+c_{2, \uparrow}^{+} c_{2, \downarrow}^{+} c_{1, \downarrow} c_{1, \uparrow}\right)
\end{aligned}
$$

where $t_{1,2}=t_{2,1}=-t, J_{1,2}^{(1,2)}=J_{2,1}^{(1,2)}=J^{(1,2)}$ and $V_{1,2}=V_{2,1}=V$. We start from the Fock basis $\left|n_{1, \uparrow}, n_{1, \downarrow} ; n_{2, \uparrow}, n_{2, \downarrow}\right\rangle\left(n_{i, \sigma}=0,1 ; i=1,2 ; \sigma=\uparrow, \downarrow\right)$ and we find the exact solution of the dimer eigenvalue problem $\left(H_{\mathrm{D}}\left|E_{\alpha}\right\rangle=E_{\alpha}\left|E_{\alpha}\right\rangle\right)$ :

$$
\begin{gathered}
E_{1}=0,\left|E_{1}\right\rangle=|0,0 ; 0,0\rangle, E_{2}=-t,\left|E_{2}\right\rangle=\frac{|1,0 ; 0,0\rangle+|0,0 ; 1,0\rangle}{\sqrt{2}}, \\
E_{3}=t,\left|E_{3}\right\rangle=\frac{|1,0 ; 0,0\rangle-|0,0 ; 1,0\rangle}{\sqrt{2}}, E_{4}=-t,\left|E_{4}\right\rangle=\frac{|0,1 ; 0,0\rangle+|0,0 ; 0,1\rangle}{\sqrt{2}}, \\
E_{5}=t,\left|E_{5}\right\rangle=\frac{(|0,1 ; 0,0\rangle-|0,0 ; 0,1\rangle)}{\sqrt{2}}, E_{6}=J^{(2)},\left|E_{6}\right\rangle=\frac{|1,0 ; 0,1\rangle+|0,1 ; 1,0\rangle}{\sqrt{2}}, \\
E_{7}=U+V,\left|E_{7}\right\rangle=\frac{|1,1 ; 0,0\rangle-|0,0 ; 1,1\rangle}{\sqrt{2}}, \\
E_{8}=C+\frac{U-V+J^{(2)}}{2}, \\
\left|E_{8}\right\rangle=a_{+}(|1,1 ; 0,0\rangle+|0,0 ; 1,1\rangle)-a_{-}(|1,0 ; 0,1\rangle-|0,1 ; 1,0\rangle) \\
E_{9}=-C+\frac{U-V+J^{(2)}}{2}
\end{gathered}
$$




$$
\begin{aligned}
& \left|E_{9}\right\rangle=a_{-}(|1,1 ; 0,0\rangle+|0,0 ; 1,1\rangle)+a_{+}(|1,0 ; 0,1\rangle-|0,1 ; 1,0\rangle), \\
& E_{10}=J^{(1)},\left|E_{10}\right\rangle=|1,0 ; 1,0\rangle, E_{11}=J^{(1)},\left|E_{11}\right\rangle=|0,1 ; 0,1\rangle, \\
& E_{12}=t+U+J^{(1)}+J^{(2)},\left|E_{12}\right\rangle=\frac{|0,1 ; 1,1\rangle+|1,1 ; 0,1\rangle}{\sqrt{2}}, \\
& E_{13}=-t+U+J^{(1)}+J^{(2)},\left|E_{13}\right\rangle=\frac{|0,1 ; 1,1\rangle-|1,1 ; 0,1\rangle}{\sqrt{2}}, \\
& E_{14}=t+U+J^{(1)}+J^{(2)},\left|E_{14}\right\rangle=\frac{|1,0 ; 1,1\rangle+|1,1 ; 1,0\rangle}{\sqrt{2}}, \\
& E_{15}=-t+U+J^{(1)}+J^{(2)},\left|E_{15}\right\rangle=\frac{|1,0 ; 1,1\rangle-|1,1 ; 1,0\rangle}{\sqrt{2}}, \\
& E_{16}=2\left(U+J^{(1)}+J^{(2)}\right), \quad\left|E_{16}\right\rangle=|1,1 ; 1,1\rangle,
\end{aligned}
$$

where

$$
C=\sqrt{\left(\frac{U-V-J^{(2)}}{2}\right)^{2}+4 t^{2}}, \quad a_{ \pm}=\frac{1}{2} \sqrt{1 \pm \frac{U-V-J^{(2)}}{2 C}} .
$$

In the following we apply the Hubbard and spin operators (cf. e.g. Ref. [1])

$$
\begin{aligned}
& a_{i, \sigma}=c_{i, \sigma}\left(1-n_{i,-\sigma}\right), \quad b_{i, \sigma}=c_{i, \sigma} n_{i,-\sigma}, \\
& S_{i}^{z}=\frac{1}{2}\left(n_{i, \uparrow}^{a}-n_{i, \downarrow}^{a}\right), \quad n_{i, \sigma}^{a}=a_{i, \sigma}^{+} a_{i, \sigma}, \\
& S_{i}^{+}=c_{i, \uparrow}^{+} c_{i, \downarrow}=a_{i, \uparrow}^{+} a_{i, \downarrow}, \quad S_{i}^{-}=c_{i, \downarrow}^{+} c_{i, \uparrow}=a_{i, \downarrow}^{+} a_{i, \uparrow}
\end{aligned}
$$

and we use the equivalent expression for the dimer Hamiltonian (2)

$$
H_{\mathrm{D}}=\sum_{\alpha=1}^{16} E_{\alpha} P_{\alpha}
$$

where $P_{\alpha}=\left|E_{\alpha}\right\rangle\left\langle E_{\alpha}\right|$. Each product $E_{\alpha} P_{\alpha}$ in the formula (7) where we insert $E_{\alpha}$ and $\left|E_{\alpha}\right\rangle$ from the formulae (3) can be rewritten in the second quantization with the use of the Hubbard and spin operators (5) and (6). It is convenient to collect all the terms which correspond to the same energy level (as e.g. $\left.E_{4}=E_{2}, E_{5}=E_{3}, E_{11}=E_{10}, E_{14}=E_{12}, E_{15}=E_{13}\right)$ and the same number of particles $N$. Using (7) we can split $H_{\mathrm{D}}$ into 10 terms, corresponding to 10 different dimer energy levels (see (3)) and belonging to different subspaces of the total number of particles $N$. We obtain

$$
H_{\mathrm{D}}=\sum_{i=1}^{10} H_{\mathrm{D}}^{(i)}
$$

where

$$
H_{\mathrm{D}}^{(1)}=E_{2} P_{2}+E_{4} P_{4}=-\frac{t}{2}\left[n_{1}^{a}\left(1-n_{2}^{a}-\frac{n_{2}^{b}}{2}\right)+n_{2}^{a}\left(1-n_{1}^{a}-\frac{n_{1}^{b}}{2}\right)\right]
$$




$$
\begin{aligned}
& -\frac{t}{2} \sum_{\sigma}\left(a_{1, \sigma}^{+} a_{2, \sigma}+a_{2, \sigma}^{+} a_{1, \sigma}\right) \\
& H_{\mathrm{D}}^{(2)}=E_{3} P_{3}+E_{5} P_{5}=\frac{t}{2}\left[n_{1}^{a}\left(1-n_{2}^{a}-\frac{n_{2}^{b}}{2}\right)+n_{2}^{a}\left(1-n_{1}^{a}-\frac{n_{1}^{b}}{2}\right)\right] \\
& -\frac{t}{2} \sum_{\sigma}\left(a_{1, \sigma}^{+} a_{2, \sigma}+a_{2, \sigma}^{+} a_{1, \sigma}\right) \\
& H_{\mathrm{D}}^{(3)}=E_{6} P_{6}=-J^{(2)}\left(S_{1}^{z} \cdot S_{2}^{z}-\frac{n_{1}^{a} n_{2}^{a}}{4}\right) \\
& +\frac{J^{(2)}}{2}\left(S_{1}^{+} \cdot S_{2}^{-}+S_{1}^{-} \cdot S_{2}^{+}\right) \\
& H_{\mathrm{D}}^{(4)}=E_{7} P_{7}=\frac{U+V}{4}\left[n_{1}^{b}\left(1-n_{2}^{a}-\frac{n_{2}^{b}}{2}\right)+n_{2}^{b}\left(1-n_{1}^{a}-\frac{n_{1}^{b}}{2}\right)\right] \\
& -\frac{U+V}{2}\left(d_{1}^{+} d_{2}+d_{2}^{+} d_{1}\right) \\
& H_{\mathrm{D}}^{(5)}=E_{8} P_{8}=\left[-\frac{J^{(2)}}{2}+\frac{J^{(2)}\left(U-V-J^{(2)}\right)}{4 C}-\frac{2 t^{2}}{C}\right]\left(\boldsymbol{S}_{1} \cdot \boldsymbol{S}_{2}-\frac{n_{1}^{a} n_{2}^{a}}{4}\right) \\
& +\left[\frac{U-V}{4}\left(1+\frac{U-V-J^{(2)}}{2 C}\right)+\frac{t^{2}}{C}\right]\left(d_{1}^{+} d_{2}+d_{2}^{+} d_{1}\right) \\
& +\left[\frac{U-V}{8}\left(1+\frac{U-V-J^{(2)}}{2 C}\right)+\frac{t^{2}}{2 C}\right] \\
& \times\left[n_{1}^{b}\left(1-n_{2}^{a}-\frac{n_{2}^{b}}{2}\right)+n_{2}^{b}\left(1-n_{1}^{a}-\frac{n_{1}^{b}}{2}\right)\right] \\
& -\frac{t}{2}\left[1+\frac{U-V+J^{(2)}}{2 C}\right] \sum_{\sigma} \sum_{i=1}^{2}\left(a_{i, \sigma}^{+} b_{\bar{i}, \sigma}+b_{i, \sigma}^{+} a_{\bar{i}, \sigma}\right), \\
& H_{\mathrm{D}}^{(6)}=E_{9} P_{9}=\left\{-\frac{J^{(2)}}{2}-\left[\frac{J^{(2)}\left(U-V-J^{(2)}\right)}{4 C}-\frac{2 t^{2}}{C}\right]\right\}\left(\boldsymbol{S}_{1} \cdot \boldsymbol{S}_{2}-\frac{n_{1}^{a} n_{2}^{a}}{4}\right) \\
& +\left[\frac{U-V}{4}\left(1-\frac{U-V-J^{(2)}}{2 C}\right)-\frac{t^{2}}{C}\right]\left(d_{1}^{+} d_{2}+d_{2}^{+} d_{1}\right) \\
& +\left[\frac{U-V}{8}\left(1-\frac{U-V-J^{(2)}}{2 C}\right)-\frac{t^{2}}{2 C}\right] \\
& \times\left[n_{1}^{b}\left(1-n_{2}^{a}-\frac{n_{2}^{b}}{2}\right)+n_{2}^{b}\left(1-n_{1}^{a}-\frac{n_{1}^{b}}{2}\right)\right] \\
& -\frac{t}{2}\left[1-\frac{U-V+J^{(2)}}{2 C}\right] \sum_{\sigma} \sum_{i=1}^{2}\left(a_{i, \sigma}^{+} b_{\bar{i}, \sigma}+b_{i, \sigma}^{+} a_{\bar{i}, \sigma}\right),
\end{aligned}
$$




$$
\begin{aligned}
& H_{\mathrm{D}}^{(7)}=E_{10} P_{10}+E_{11} P_{11}=2 J^{(1)}\left(S_{1}^{z} \cdot S_{2}^{z}+\frac{n_{1}^{a} n_{2}^{a}}{4}\right) \\
& H_{\mathrm{D}}^{(8)}=E_{12} P_{12}+E_{14} P_{14}=\frac{t+U+J^{(1)}+J^{(2)}}{4}\left(n_{1}^{a} n_{2}^{b}+n_{2}^{a} n_{1}^{b}\right) \\
& \quad-\frac{t+U+J^{(1)}+J^{(2)}}{2} \sum_{\sigma}\left(b_{1, \sigma}^{+} b_{2, \sigma}+b_{2, \sigma}^{+} b_{1, \sigma}\right), \\
& \quad+\frac{-t+U+J^{(1)}+J^{(2)}}{2} \sum_{\sigma}^{(9)}\left(b_{1, \sigma}^{+} b_{2, \sigma}+b_{2, \sigma}^{+} b_{1, \sigma}\right) \\
& H_{\mathrm{D}}^{(10)}=E_{13} P_{13}+E_{15} P_{15}=\frac{-t+J^{(1)}+J^{(2)}}{4}\left(n_{1}^{a} n_{2}^{b}+n_{2}^{a} n_{1}^{b}\right) \\
& 2=\frac{U+J^{(1)}+J^{(2)}}{2} n_{1}^{b} n_{2}^{b},
\end{aligned}
$$

and $n_{i}^{a, b}=n_{i, \uparrow}^{a, b}+n_{i, \downarrow}^{a, b}, n_{i, \sigma}^{b}=b_{i, \sigma}^{+} b_{i, \sigma}=n_{i, \sigma} n_{i,-\sigma}(i=1,2), d_{1(2)}=a_{1(2), \downarrow} b_{1(2), \uparrow}=$ $c_{1(2), \downarrow} c_{1(2), \uparrow}, \bar{i}=1$ if $i=2$ and $\bar{i}=2$ if $i=1$. The partial Hamiltonians $((9),(10)),((11)-(15)),((16),(17))$, and (18) belong to the subspaces of $N=$ $1,2,3$, and 4 , respectively. The expressions (9)-(18) are exact and when we sum them up (see (8)) we obtain again the dimer Hamiltonian in the form given by the expression (2), as it should be. The decomposition (8) of the dimer Hamiltonian (2) into 10 different parts (9)-(18) according to dimer energy levels possesses several, important advantages. First, it explicitly visualizes the important intrinsic two-site interactions, deeply hidden in the dimer Hamiltonian (2). Due to the fact that the formulae (9)-(18) are exact the information about the competitive interactions within the model for a dimer is complete. Second, all of them are ascribed to each dimer energy level. It, however, means that such interactions can be thermodynamically active only in the case when the corresponding level will be occupied by electrons. Third, we can see that the same types of interactions (but with different coupling constants) belong to quite different energy levels. It also means that they do not need to be thermodynamically active at the same time (it depends on the occupation of the particular levels) and that the resulting properties of the system depend on their competition. Fourth, the formulae (9)-(18) visualize the important fact that with the increase in the averaged number of electrons $n=\langle N\rangle$ the system will pass through different phases, depending on the result of the competition between different thermodynamically activated two-sites interactions.

The most important two-site intrinsic interactions (leading to magnetism or superconductivity), presented in the expressions (9)-(18) can be divided into two classes. First of them belongs to magnetic interactions (ferromagnetic, antiferromagnetic - it depends on the sign of the parameters $J^{(1)}, J^{(2)}, U$, and $V$ ). Such interactions are present in the formulae (11) and (15) and describe Ising-type in- 
teractions with coupling constants generated by $J^{(1)}$ and $J^{(2)}$. The formula (11) contains also the transverse interaction between spins. The Heisenberg-type magnetic interactions can be seen in the first terms of the formulae (13) and (14), generated by more complex coupling constants, expressed by the model parameters $J^{(2)}, U, V$, and $t$. It is interesting to note that when $J^{(2)}=V=0$ the first term in the formulae (13) and (14) describes ferromagnetic or antiferromagnetic interactions, similar to the well-known $t-J$ model (see e.g. Refs. [65-75, 89]) because the coefficient $2 t^{2} / C \approx 4 t^{2} /|U|$ for large $|U|$ and is exactly the same as in the case of a real lattice. The first terms in the expressions (13) and (14) can also be considered as generalized $t-J$ interactions, valid in a more general case of the model (2). The coupling constants in the first terms in (13) and (14) can be negative or positive. Thus, they can describe competitive ferromagnetic or antiferromagnetic Heisenberg interactions belonging to different dimer energy levels. In the case of the conventional Hubbard model $\left(J^{(1)}=J^{(2)}=V=0\right)$ the coupling constants in the first terms in (13) and (14) are reduced to $-2 t^{2} / C$ (13) and $2 t^{2} / C$ (14), which means that the conventional Hubbard model for a dimer describes ferromagnetic and antiferromagnetic Heisenberg interactions which compete together, but they belong to different dimer energy levels. Let us note that the terms containing the products like $d_{1}^{+} d_{2}=b_{1, \uparrow}^{+} a_{1, \downarrow}^{+} a_{2, \downarrow} b_{2, \uparrow}=c_{1, \uparrow}^{+} c_{1, \downarrow}^{+} c_{2, \downarrow} c_{2, \uparrow}$ and $d_{2}^{+} d_{1}=b_{2, \uparrow}^{+} a_{2, \downarrow}^{+} a_{1, \downarrow} b_{1, \uparrow}=$ $c_{2, \uparrow}^{+} c_{2, \downarrow}^{+} c_{1, \downarrow} c_{1, \uparrow}$ describe the hopping of the Cooper pairs. Such terms, present in the second terms in (12), (13), and (14) are typical of the KPPK superconductivity models (cf. e.g. Refs. [59-64]) with positive or negative coupling constants. Let us, however, note that this type of interactions are also present within the model (2) also in the case $V=0$. The transversal products $S_{1}^{+} S_{2}^{-}\left(S_{1}^{-} S_{2}^{+}\right)$are present in the formulae (11), (13), and (14). When using the second quantization we obtain $S_{1}^{+} S_{2}^{-}=-c_{1, \uparrow}^{+} c_{2, \downarrow}^{+} c_{1, \downarrow} c_{2, \uparrow}\left(S_{1}^{-} S_{2}^{+}=-c_{2, \uparrow}^{+} c_{1, \downarrow}^{+} c_{2, \downarrow} c_{1, \uparrow}\right)$ and these terms describe intersite Cooper pairs (cf. e.g. Ref. [33] and papers cited therein). The application of the resonating valence bond approach (cf. Refs. [28, 29, 52]) allows us also to introduce the pairing operator $f_{2,1}=\left(c_{2, \downarrow} c_{1, \uparrow}-c_{2, \uparrow} c_{1, \downarrow}\right) / \sqrt{2}$ and e.g. when we restrict ourselves to the subspace $n_{1}^{a}=n_{2}^{a}=1$ we can represent the $t-J$ terms in (13) and (14) in the following way: $\left(\boldsymbol{S}_{1} \cdot \boldsymbol{S}_{2}-\frac{1}{4}\right)=-f_{2,1}^{+} f_{2,1}$ and such terms lead to superconductivity (cf. Ref. [29]). It is also interesting to note that even in the case when $J^{(1)}=J^{(2)}=U=V=0$ (see formulae (9)-(18)) the main competitive interactions (ferromagnetic, antiferromagnetic, and superconducting) are always present, when only the hopping parameter does not vanish $(t \neq 0)$.

\section{Conclusions}

Let us consider the case of a real lattice described by the Hamiltonian (1). We assume that the number of lattice points $i(j)$ is equal to $N$ (even number) and we decompose the lattice into a set of $M=N / 2$ dimers described by the dimer index $I, \alpha(J, \beta)$ where $I(J)=1,2, \ldots, M$ and $\alpha(\beta)=1,2$. The Hamiltonian (1) can thus be replaced by the equivalent form 


$$
\begin{aligned}
H= & \sum_{I} H_{\mathrm{D}, I}+\sum_{I \neq J, \alpha, \sigma} t_{I, \alpha ; J, \alpha} c_{I, \alpha, \sigma}^{+} c_{J, \alpha, \sigma}+\sum_{I \neq J, \alpha \neq \beta, \sigma} t_{I, \alpha ; J, \beta} c_{I, \alpha, \sigma}^{+} c_{J, \beta, \sigma} \\
& +\frac{1}{2} \sum_{I \neq J, \alpha, \sigma} J_{I, \alpha ; J, \alpha}^{(1)} n_{I, \alpha, \sigma} n_{J, \alpha, \sigma}+\frac{1}{2} \sum_{I \neq J, \alpha \neq \beta, \sigma} J_{I, \alpha ; J, \beta}^{(1)} n_{I, \alpha, \sigma} n_{J, \beta, \sigma} \\
& +\frac{1}{2} \sum_{I \neq J, \alpha, \sigma} J_{I, \alpha ; J, \alpha}^{(2)} n_{I, \alpha, \sigma} n_{J, \alpha,-\sigma}+\frac{1}{2} \sum_{I \neq J, \alpha \neq \beta, \sigma} J_{I, \alpha ; J, \beta}^{(2)} n_{I, \alpha, \sigma} n_{J, \beta,-\sigma} \\
& -\sum_{I \neq J, \alpha} V_{I, \alpha ; J, \alpha} c_{I, \alpha, \uparrow}^{+} c_{I, \alpha, \downarrow}^{+} c_{J, \alpha, \downarrow} c_{J, \alpha, \uparrow} \\
& -\sum_{I \neq J, \alpha \neq \beta} V_{I, \alpha ; J, \beta} c_{I, \alpha, \uparrow}^{+} c_{I, \alpha, \downarrow}^{+} c_{J, \beta, \downarrow} c_{J, \beta, \uparrow},
\end{aligned}
$$

where $H_{\mathrm{D}, I}$ is the dimer Hamiltonian given by the expression (2), where the lower dimer index $I$ in the operators should be introduced (as e.g. $c_{1, \sigma} \rightarrow c_{I, 1, \sigma}$, etc.). The dimer Hamiltonian $H_{\mathrm{D}, I}$ in (19) can, however, be diagonalized and replaced by the expression (8), where again the dimer index $I$ in the operators appearing in the expressions (9)-(18) should be introduced. The Hamiltonian (19), equivalent to (1), describes now "free" dimers (the first term in (19)) and their interactions (the next 8 terms in (19)). It is then evident that the Hamiltonian (19) contains explicitly all the competitive two-site interactions present in the dimer Hamiltonian alone (see the formulae (8) and (9)-(18)) but supplemented by the dimer interactions, represented by the second and further terms in (19). The main difference between Hamiltonians (1) and (19), both describing the same physics, lies in the fact that the above-mentioned competitive magnetic and superconducting interactions are hidden in the Hamiltonian (1), whereas in the Hamiltonian (19) they appear now in a direct way. It, however, means that to find thermodynamical properties of the model we should simultaneously introduce order parameters in four competitive channels (ferromagnetic, antiferromagnetic, and superconducting (intrasite and intersite pairing)), where a special attention should be paid to the nontrivial fact that all competitive interactions are additionally scattered between different energy levels and therefore, their activities have to be correlated with the occupation of these levels.

\section{References}

[1] J. Hubbard, Proc. R. Soc. A 276, 238 (1963); 281, 401 (1964).

[2] M.C. Gutzwiller, Phys. Rev. Lett. 10, 59 (1963).

[3] J. Kanamori, Prog. Theor. Phys. 30, 275 (1963).

[4] C. Herring, in: Magnetism, Vol. IV, Eds. G.T. Rado, H. Suhl, Academic Press, New York 1966, p. 1.

[5] J. Friedel, in: The Physics of Metals, Ed. J.M. Ziman, Cambridge University Press, Cambridge 1969, p. 340.

[6] B. Brandow, Adv. Phys. 26, 651 (1977).

[7] T. Moriya, Spin Fluctuations in Itinerant Electron Magnetism, Springer Series in Solid State Sciences, Vol. 56, Springer, Berlin 1985. 
[8] W. Nolting, Quantentheorie des Magnetismus, Vol. 1 and 2, Teubner, Stuttgart 1986.

[9] Metallic Magnetism, Ed. H. Capelmann, Springer Topics in Current Physics, Vol. 42, Springer, Berlin 1987.

[10] N.F. Mott, Metal-Insulator Transitions, Taylor and Francis, London 1990.

[11] P.A. Cox, Transition Metal Oxides, International Series of Monographs on Chemistry, Vol. 27, Clarendon Press, Oxford 1992.

[12] D.E. Logan, Y.H. Szczech, M.E. Tusch, in: Metal-Insulator Transitions Revisited, Eds. P.P. Edwards, C.N.R. Rao, Taylor and Francis, London 1995, p. 395.

[13] P. Fulde, Electron Correlations in Molecules and Solids, Springer, Berlin 1995.

[14] T. Herrmann, W. Nolting, J. Magn. Magn. Mater. 170, 253 (1997).

[15] F. Gebhard, The Mott Metal-Insulator Transitions, Springer Tracts in Modern Physics, Vol. 137, Springer, Berlin 1997.

[16] M. Imada, A. Fujimori, Rev. Mod. Phys. 70, 1039 (1998).

[17] P.W. Anderson, Phys. Rev. 124, 41 (1961).

[18] C. Varma, Y. Yafet, Phys. Rev. B 13, 2950 (1976).

[19] D. Vollhardt, Rev. Mod. Phys. 56, 99 (1984).

[20] D. Vollhardt, P. Woelfle, P.W. Anderson, Phys. Rev. B 35, 6703 (1987).

[21] D. Vollhardt, P. Woelfle, The Superfluid Phases of Helium-3, Taylor and Francis, London 1990.

[22] Electronic Properties of Fullerenes, Eds. H. Kuzmany, J. Fink, M. Mehring, S. Roth, Springer Series in Solid State Sciences, Vol. 117, Springer, Berlin 1993.

[23] S. Chakravarty, M.P. Gelfand, S. Kivelson, Science 254, 970 (1991).

[24] S.L. Sondhi, M.P. Gelfand, H.Q. Lin, D.K. Campbell, Phys. Rev. B 51, 593 (1995).

[25] S. Robaszkiewicz, R. Micnas, K.A. Chao, Phys. Rev. B 23, 1447 (1981); 26, 3915 (1982).

[26] J. Ranninger, S. Robaszkiewicz, Physica B 135, 486 (1985).

[27] A.S. Alexandrov, J. Ranninger, S. Robaszkiewicz, Phys. Rev. B 33, 4526 (1986).

[28] P.W. Anderson, Science 235, 1196 (1987).

[29] G. Baskaran, P.W. Anderson, Phys. Rev. B 37, 580 (1988).

[30] R. Micnas, J. Ranninger, S. Robaszkiewicz, Phys. Rev. B 37, 9410 (1988); B 39, 11653 (1989).

[31] G.T. Zimanyi, S.A. Kivelson, A. Luther, Phys. Rev. Lett. 60, 2089 (1988).

[32] M. Inui, S. Doniach, P.J. Hirschfeld, A.E. Rueckenstein, Phys. Rev. B 37, 2320 (1988).

[33] R. Micnas, J. Ranninger, S. Robaszkiewicz, Rev. Mod. Phys. 62, 113 (1990).

[34] P.G.J. van Dongen, Phys. Rev. Lett. 67, 757 (1991).

[35] G.A. Lara, G.G. Cabrera, Phys. Rev. B 47, 14417 (1993).

[36] Y.Z. Yan, Phys. Rev. B 48, 7140 (1993).

[37] E. Dagotto, Rev. Mod. Phys. 66, 763 (1994). 
[38] P.G.J. van Dongen, Phys. Rev. B 50, 14031 (1994).

[39] A.B. Eriksson, T. Einarson, S. Ostlund, Phys. Rev. B 52, 3662 (1995).

[40] B.R. Bułka, S. Robaszkiewicz, Phys. Rev. B 54, 13138 (1996).

[41] H. Tasaki, J. Phys. C 10, 4378 (1998).

[42] G.I. Japaridze, A.P. Kampf, Phys. Rev. B 59, 12822 (1999).

[43] R. Micnas, S. Robaszkiewicz, B. Tobijaszewska, J. Supercond. 12, 79 (1999).

[44] G.I. Japaridze, E. Müller-Hartmann, Phys. Rev. B 61, 113 (2000).

[45] J.X. Zhu, C.S. Ting, Phys. Rev. B 61, 1456 (2000).

[46] M. Imada, M. Kohno, Phys. Rev. Lett. 84, 143 (2000).

[47] G.I. Japaridze, A.P. Kampf, M. Sekania, P. Kakashvilli, B. Brune, Phys. Rev. B 65, 14518 (2001).

[48] J. Mizia, G. Górski, K. Kucab, Phys. Status Solidi B 229, 1221 (2002).

[49] R. Micnas, S. Robaszkiewicz, A. Bussmann-Holder, Phys. Rev. B 66, 104516 (2002).

[50] R. Micnas, B. Tobijaszewska, J. Phys. C 14, 9631 (2002).

[51] G. Baskaran, Phys. Rev. Lett. 91, 097003 (2003).

[52] C. Dziurzik, G.I. Japaridze, A. Schadschneider, J. Zittartz, Eur. Phys. J. B 37, 453 (2004).

[53] W.R. Czart, S. Robaszkiewicz, Acta Phys. Pol. A 106, 709 (2004).

[54] M. Bak, Phys. Status Solidi B 242, 420 (2005).

[55] B. Tobijaszewska, R. Micnas, Phys. Status Solidi B 242, 468 (2005).

[56] W.R. Czart, S. Robaszkiewicz, Phys. Status Solidi B 243, 151 (2006).

[57] W.R. Czart, S. Robaszkiewicz, Acta Phys. Pol. A 109, 577 (2006).

[58] I.O. Kulik, A.G. Pedan, Zh. Eksp. Teor. Fiz. 79, 1469 (1980).

[59] K.A. Penson, M. Kolb, Phys. Rev. B 33, 1663 (1986).

[60] S. Robaszkiewicz, B.R. Bułka, Phys. Rev. B 59, 6430 (1999).

[61] M. Nakamura, Phys. Rev. B 61, 16377 (2000).

[62] W.R. Czart, S. Robaszkiewicz, Phys. Rev. B 64, 104511 (2001).

[63] M. Mierzejewski, M. Maśka, Phys. Rev. B 69, 054502 (2004).

[64] P.W. Anderson, Phys. Rev. 115, 2 (1959).

[65] P.W. Anderson, in: Solid State Physics, Eds. F. Seitz, D. Turnbull, Vol. 14, Academic Press, New York 1963, p. 99.

[66] D.J. Klein, W.A. Seitz, Phys. Rev. B 8, 2236 (1973).

[67] J. Florencio, Jr, K.A. Chao, Phys. Rev. Lett. 35, 741 (1975).

[68] W. Kohn, Phys. Rev. 133, 171 (1964).

[69] A.B. Harris, R.V. Lange, Phys. Rev. 157, 295 (1967).

[70] T.A. Kaplan, R.A. Bari, J. Appl. Phys. 41, 875 (1970).

[71] J. Florencio, Jr, K.A. Chao, Phys. Rev. B 14, 3121 (1976).

[72] K.A. Chao, J. Spałek, A.M. Oleś, J. Phys. C 10, L271 (1977); Phys. Status Solidi B 84, 747 (1977); Phys. Rev. B 18, 3453 (1980). 
[73] J. Spałek, A.M. Oleś, Physica B 86-88, 375 (1977).

[74] J. Spałek, K.A. Chao, J. Phys. C 13, 5241 (1980).

[75] V.C. Cheng, S.H. Chen, Physica B 85, 299 (1977).

[76] M. Matlak, Phys. Status Solidi B 99, K87 (1980).

[77] B. Lorenz, Phys. Status Solidi B 119, 555 (1983).

[78] L.M. Falicov, R.H. Victor, Phys. Rev. B 30, 1695 (1984).

[79] J. Callaway, D.P. Chen, R. Tang, Phys. Rev. B 35, 3705 (1987).

[80] J. Callaway, D.P. Chen, Y. Zhang, Phys. Rev. B 36, 2084 (1987).

[81] S. Sachdev, R.N. Blatt, Phys. Rev. B 41, 9323 (1990).

[82] G.M. Pastor, R. Hirsch, B. Mühlschlegel, Phys. Rev. B 53, 10382 (1996).

[83] D. Senechal, D. Perez, M. Pioto-Ladriere, Phys. Rev. Lett. 84, 522 (2000).

[84] K. Park, S. Sachdev, Phys. Rev. B 64, 184510 (2001).

[85] E.H. Lieb, F.Y. Wu, Phys. Rev. Lett. 20, 1445 (1968).

[86] A. Klümper, A. Schadschneider, J. Zittartz, Europhys. Lett. 24, 293 (1993).

[87] R. Strack, D. Vollhardt, Phys. Rev. Lett. 70, 2637 (1993).

[88] J. de Boer, A. Schadschneider, Phys. Rev. Lett. 75, 4298 (1995).

[89] A. Montorsi, D.K. Campbell, Phys. Rev. B 53, 5153 (1996).

[90] Z. Szabo, Phys. Rev. B 59, 10007 (1999).

[91] C. Dziurzik, A. Schadschneider, J. Zittartz, Eur. Phys. J. B 12, 109 (1999).

[92] M. Beccaria, Eur. Phys. J. B 14, 523 (2000).

[93] B. Grabiec, M. Matlak, Acta Phys. Pol. A 101, 537, 549 (2002).

[94] M. Matlak, J. Aksamit, B. Grabiec, W. Nolting, Ann. Phys. 12, 304 (2003).

[95] E.C. Carter, A.J. Schofield, Phys. Rev. B 70, 045107 (2004).

[96] M. Matlak, T. Słomska, B. Grabiec, Phys. Status Solidi B 242, 317, 337 (2005).

[97] D. Bimberg, M. Grundmann, N.N. Ledentsov, Quantum Dot Heterostructures, Wiley, New York 1999.

[98] D. Loos, D.P. Di Vincenzo, Phys. Rev. A 57, 120 (1998).

[99] J.I. Martin, J. Nogues, K. Liu, J.L. Vicent, I.K. Schuler, J. Magn. Magn. Mater. 256, 449 (2003).

[100] F. Baletto, R. Ferrando, Rev. Mod. Phys. 77, 371 (2004). 